www.jmscr.igmpublication.org

Index Copernicus Value: 79.54

ISSN (e)-2347-176x ISSN (p) 2455-0450

crossref DOI: https://dx.doi.org/10.18535/jmscr/v7i6.14

\title{
Type I Spinal Muscular Atrophy (Werdnig-Hoffman Disease) - A Rare Neurodegenerative Disorder in Infancy
}

\author{
Authors \\ Parveen Bhardwaj, Champa Panwar, Neeraj Sharma
}

\begin{abstract}
Childhood spinal muscular atrophy is an autosomal recessive neuromuscular disease characterized by degeneration of the anterior horn cells of the spinal cord. SMA type I or Werdnig-Hoffmann disease (WHD), the most severe form can be detected in utero or during the first months of life. Death typically occurs within the first 2 years of life. A 6-month old male was admitted to the emergency room for severe respiratory distress. He had generalised hypotonia, symmetrical proximal muscle weakness, areflexia, intercostal muscle weakness and tongue fasciculations. Molecular analysis reported homozygous deletion of exons 7 of I. It is imperative to recognize and diagnose this entity in order to provide genetic counselling to the family as well as to offer support and advice in the care of the patient.

Keywords: Autosomal recessive, degenerative disorder, areflexia, fasciculations, genetic conselling.
\end{abstract}

\section{Introduction}

Spinal muscular atrophies (SMAs) are a rare heterogenous group of genetic degenerative diseases of motor neurons that begin in fetal life and continue to be progressive in infancy and childhood. It is one of the leading genetic causes of death in infancy after cystic fibrosis. ${ }^{1}$ We report the case of a 6 month infant with WerdnigHoffmann disease, most severe form of SMA with a high mortality in first two years of life.

We present the case of a 6 months old male infant, admitted to PICU with the complaints of cough and fast breathing for 7 days, fever and decreased acceptance of feeds for 2 days.He was a product non - consanguineous marriage with uneventful antenatal period. Born by full term vaginal delivery at hospital with a birth weight of $3 \mathrm{~kg}$, Apgar was unknown. There was history of gross and fine motor delay whereas development in other spheres was normal. The infant had no significant past and family history. At admission the infant presented with respiratory distress. There were eczematous lesions all over the body. Chest was bell shaped and auscultation revealed bronchial breathing over right side of chest and bilateral crepts. Cardiovascular and abdominal examination was normal. Central nervous system examination was suggestive of generalised hypotonia, symmetrical proximal muscle weakness, areflexia, frog like posture, fasciculations of tongue and normal fundus (figure 1). There were no meningeal signs, dysmorphism or congenital anomalies. 


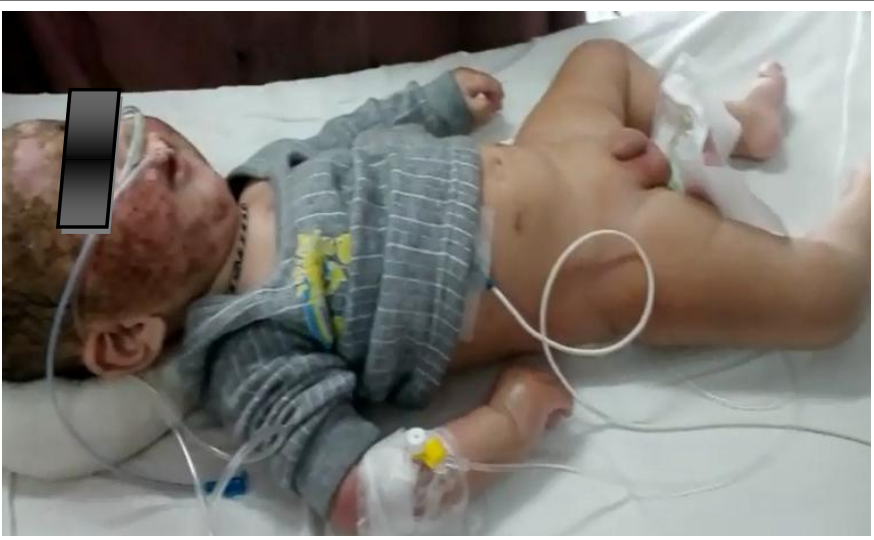

Figure 1. Infant had eczematous lesions over face, frog like posture, hypotonia of all four extremities with characteristic position: arms extended along the trunk, forearms pronated and lower limbs abducted, externally rotated and resting on the examination table.

Random blood sugar, renal and liver function tests, electrolytes, serum creatinine kinase, Thyroid function tests, arterial blood gas analysis were normal and tuberculosis workup was negative.Complete blood counts revealed Neutrophilic leucocytosis.ESR was raised. Chest $\mathrm{X}$ - ray was suggestive of pneumonia right lung.In view of above mentioned neurological findings a strong possibility of Floppy infant with SMA was kept and blood sample for SMN gene mutation analysis was also sent. Electromyography and Nerve conduction Velocity studies could not be performed in our case.

Patient received treatment consisting of oxygen with nasal prongs, bronchodilators, maintenance fluids, antibiotics, orogastric feeds, and physiotherapy. Genetic counseling of the parents was done. On day 15 of admission patient developed respiratory failure and he was planned for ventilatory support. His condition and poor prognosis was discussed with the attendants but they refused for further continuation of treatment and opted for domiciliary care. Molecular genetic analysis of the patient revealed homozygous deletion of exon 7 of SMN1 gene and hence confirmed our diagnosis of Type 1SMA.

WHD is the most severe form of childhood SMA and manifests itself during the first months of life as a pathological continuation of programmed cell death. Incidence is .5 to 1 per 25,000 live births and affects all ethnic groups. ${ }^{1}$ It is the second most common neuromuscular disease, following Duchenne muscular dystrophy. ${ }^{2}$ The progressive denervation of muscle is compensated in part by reinnervation from an adjacent motor unit, but giant motor units are thus created with subsequent atrophy of muscle fibers. Upper motor neurons remain normal. $^{3}$

Diagnostic suspicion is mainly clinical like it was in our case, therefore, childhood muscle atrophy should be suspected while examining an infant with marked hypotonia. ${ }^{4}$ It is important to consider that the hypotonia may go unnoticed by the family or the infant may present with developmental delay. The cause for hospitalization may be respiratory failure as in the case presented here, which can be mistaken for pneumonia if proper questioning and an adequate physical examination are not performed. During the study of a hypotonic infant differential diagnoses may be metabolic diseases such as glycogen storage disease, hypothyroidism, poisoning (botulism) and congenital neuromuscular diseases should also be kept. ${ }^{5}$

SMN gene containing 8 exons arrests apoptosis of motor neuroblasts. $S M N$-lgene deletion at chromosome 5 at the $5 \mathrm{q} 11-\mathrm{q} 13$ locus is seen in $95 \%$ of patients. ${ }^{2}$ Isolated mutations or deletions of the neuronal apoptosis inhibitory gene (NAIP) generate a nonfunctional isoform lacking the carboxy-terminus amino acids encoded by exon 7 and results in milder forms of SMA. Molecular genetic diagnosis using $S M N$ gene mutation in blood samples, muscle biopsy or chorionic villi tissues is gold standard for diagnosis of suspected cases, carriers as well as prenatal diagnosis. ${ }^{6,7}$ The result of the molecular study allows appropriate genetic counselling for the family because it indicates a more than $99 \%$ probability that the biological parents of the patient are 8heterozygous or healthy carriers. Because SMA has an autosomal recessive inheritance, the risk of disease recurrence in the offspring of parents, regardless of gender, is $25 \%$ with each 
pregnancy. ${ }^{8,9}$ Serum CK level may be normal or elevated. NCV is normal except for mild slowing in terminal stages of the disease. EMG is suggestive of fibrillation, denervation potentials, high amplitude waves and motor unit potentials of long duration with a reduced recruitment pattern. ${ }^{8,9,10}$ Muscle biopsy shows groups of giant type I fibers mixed with fascicles of severely atrophic fibers of both histochemical types. ${ }^{10}$

\section{Conclusion}

SMA should be kept in mind in the differential diagnosis for unexplained severe generalized hypotonia and respiratory distress immediately after birth in the neonates. Till date no medical treatment is able to delay the progression. Supportive therapy in the form of multidisciplinary approach includes orthopaedic care, physiotherapy, prevention and management of complications.

\section{Acknowledgement}

Parents for their consent for the publication.

\section{References}

1. Monani UR. Spinal muscular atrophy: a deficiency in a ubiquitous protein; a motor neuron-specifi c disease. Neuron 2005; 48:885-896.

2. Melki J, Abdelhak S, Sheth P, Bachelot MF, Burlet P, Marcadet A, et al. Gene for chronic proximal spinal muscular atrophies maps to chromosome 5q. Nature 1990; 344:767-768.

3. Oskoui, M.; Levy, G.; Garland, C. J.; Gray, J. M.; O'Hagen, J.; De Vivo, D. C.; Kaufmann, P. : The changing natural history of spinal muscular atrophy type 1 . Neurology 2007; 69: 1931-1936.

4. Dubowitz V. Muscle Disorders of Childhood. Philadelphia Saunders; 1978. pp. 146-190.
5. Thomas, N. H.; Dubowitz, V: The natural history of type I (severe) spinal muscular atrophy. Neuromusc. Disord. 2007; 4: 497 502, 1994.

6. Burlet $\mathrm{P}$, Bürglen L, Clermont $\mathrm{O}$, Lefebvre S, Viollet L, Munnich A, et al. Large scale deletions of the $5 q 13$ region are specific to Werdnig-Hoffmann disease. J Med Genet 1996; 33:281-283.

7. Scheffer H, Cobben JM, Matthijs G, Wirth B.:Best practice guidelines for molecular analysis in spinal muscular atrophy. Eur $\mathbf{J}$ Hum Genet 2001; 9:484-49.

8. Wirth, B.; Rudnik-Schoneborn, S.; Hahnen, E.; Rohrig, D.; Zerres, K.: Prenatal prediction in families with autosomal recessive proximal spinal muscular atrophy (5q11.2-q13.3): molecular genetics and clinical experience in 109 cases. Prenatal Diag. 1995; 15: 407 417.

9. Lunn MR, Wang CH. Spinal muscular atrophy. Lancet 2008; 371:2120-2133

10. Lefebvre $S$, Bürglen L, Reboullet $S$, Clermont O, Burlet P, Viollet L, et al.: Identification and characterization of a spinal muscular atrophy-determining gene. Cell 1995; 80:155-165. 